\title{
Heschl's gyrus fiber intersection area: a new insight on the connectivity of the auditory-language hub
}

\author{
Leticia Fernández, MD, ${ }^{1}$ Carlos Velásquez, MD, ${ }^{2}$ Juan A. García Porrero, MD, PhD, ${ }^{3}$ \\ Enrique Marco de Lucas, MD, ${ }^{4}$ and Juan Martino, MD, PhD ${ }^{2}$
}

\begin{abstract}
1'Department of Neurological Surgery, Hospital Universitario Araba—Santiago Apóstol, Vitoria, Spain; ${ }^{2}$ Department of Neurological Surgery, Hospital Universitario Marqués de Valdecilla and Fundación Instituto de Investigación Marqués de Valdecilla (IDIVAL), Santander, Spain; ${ }^{3}$ Department of Anatomy and Cellular Biology of Cantabria University-Medical School, Santander, Spain; and 4Department of Radiology, Hospital Universitario Marqués de Valdecilla and Fundación Instituto de Investigación Marqués de Valdecilla (IDIVAL), Santander, Spain
\end{abstract}

\begin{abstract}
OBJECTIVE The functional importance of the superior temporal lobe at the level of Heschl's gyrus is well known. However, the organization and function of these cortical areas and the underlying fiber tracts connecting them remain unclear. The goal of this study was to analyze the area formed by the organization of the intersection of Heschl's gyrusrelated fiber tracts, which the authors have termed the "Heschl's gyrus fiber intersection area" (HGFIA).

METHODS The subcortical connectivity of Heschl's gyrus tracts was analyzed by white matter fiber dissection and by diffusion tensor imaging tractography. The white matter tracts organized in relation to Heschl's gyrus were isolated in 8 human hemispheres from cadaveric specimens and in $8 \mathrm{MRI}$ studies in 4 healthy volunteers. In addition, these tracts and their functions were described in the surgical cases of left temporal gliomas next to the HGFIA in 6 patients who were awake during surgery and underwent intraoperative electrical stimulation mapping.
\end{abstract}

RESULTS Five tracts were observed to pass through the HGFIA: the anterior segment of the arcuate fasciculus, the middle longitudinal fasciculus, the acoustic radiation, the inferior fronto-occipital fasciculus, and the optic radiation. In addition, $\cup$ fibers originating at the level of Heschl's gyrus and heading toward the middle temporal gyrus were identified.

CONCLUSIONS This investigation of the HGFIA, a region where 5 fiber tracts intersect in a relationship with the primary auditory area, provides new insights into the subcortical organization of Wernicke's area. This information is valuable when a temporal surgical approach is planned, in order to assess the surgical risk related to language disturbances.

https://thejns.org/doi/abs/10.3171/2019.11.FOCUS19778

KEYWORDS arcuate fasciculus; DTI tractography; fiber dissection; Heschl's gyrus; middle longitudinal fasciculus; Wernicke's area

$\mathrm{H}$ ISTORICALLY, since Wernicke's description of the "aphasic symptom complex" in $1874,{ }^{29}$ the posterior segment of the superior temporal gyrus (STG) in the dominant hemisphere has been recognized as a key element in language processing. This area is not only important for language comprehension, but more importantly, it has a crucial role in speech production, phonologic retrieval, and semantic processing. ${ }^{1}$

Heschl's gyrus is a major component of the posterior portion of the STG and forms the anatomical substrate of the primary auditory area. The unique trajectory of the acoustic radiation from the middle geniculate body to the cortex of Heschl's gyrus has been well described. ${ }^{10,17}$ Virtually all language models emphasize the importance of the connectivity between the primary auditory area and the dorsal language stream. In fact, in the dual-stream hypothesis for language, these connections have a crucial role in processing auditory speech signals to allow articu-

ABBREVIATIONS A-Arc = anterior segment of the arcuate fasciculus; AR = acoustic radiation; D1 = shortest distance between the temporal pole and the anterior limit of Heschl's gyrus; D2 = shortest distance between the temporal pole and the anterior limit of Heschl's gyrus at the inferior limiting sulcus of the insula; D3 = shortest distance between the limen insulae and Heschl's gyrus at the inferior limiting sulcus of the insula; DTI = diffusion tensor imaging; HGFIA = Heschl's gyrus fiber intersection area; IFOF = inferior fronto-occipital fasciculus; L-Arc = long segment of the arcuate fasciculus; MLF = middle longitudinal fasciculus; $M T G=$ middle temporal gyrus; OR = optic radiation; $\mathrm{ROI}=$ region of interest; $\mathrm{STG}=$ superior temporal gyrus. 
lation and speech output. ${ }^{9}$ Numerous authors have considered the arcuate fasciculus as the main connection mediating this function, as it directly connects the temporal and frontal lobes., ${ }^{4,13,17,21}$ However, many important questions remain concerning the specific organization of these connections at the temporal lobe and the exact route out from the primary auditory area to the parietal and frontal lobes.

In the present study, the relationships of these tracts were analyzed, including a fiber intersection area at the level of the posterior area of the superior temporal lobe, around Heschl's gyrus and the acoustic radiation. We have named this area the "Heschl's gyrus fiber intersection area" (HGFIA). We combined cortex-sparing fiber dissection and diffusion tensor imaging (DTI) tractography to perform a detailed anatomical description of the topography of these neighboring tracts and their relationships. In this sense, the HGFIA anatomical organization described here adds new functional insights to the current knowledge of the role of this area in language processing. The importance of this region for the planning of temporal approaches to surgery is also discussed.

\section{Methods \\ Cortex-Sparing Fiber Dissection}

A total of 8 human cerebral hemispheres (4 left sides and 4 right sides; mean age of the subjects 73 years, range 63-82 years) were dissected by J.M. between October 2018 and November 2018. The methodology used was previously described by the author's group..$^{15,17,18,20}$

Briefly, a detailed study of the sulcal and gyral anatomy was performed in each specimen. Heschl's gyrus and the angular and supramarginal gyri, among others, were identified based on previous anatomical descriptions. ${ }^{27,28}$ Other relevant surface landmarks, such as the limen insulae, the planum polare, and the planum temporale, were also noted. All of the bundles of fibers with cortical terminations in Heschl's gyrus or crossing the subcortical area underneath were isolated, including the anterior segment of the arcuate fasciculus (A-Arc), the long segment of the arcuate fasciculus (L-Arc), the middle longitudinal fasciculus (MLF), the optic radiation (OR), the inferior fronto-occipital fasciculus (IFOF), and the acoustic radiation. The acoustic radiation was approached by starting the dissection from the posterior third of the superior temporal sulcus as described by Sarubbo et al. ${ }^{23}$ whereas the rest of the fascicles (the A-Arc, MLF, OR, and IFOF) were dissected according to the method described previously by our group. ${ }^{16}$

\section{DTI Tractography Analysis}

Brain MRI studies obtained from 4 healthy volunteers (mean age 36 years) were included in the analysis. These studies were performed with a whole-body 3.0-T scanner (Achieva 3.0T; Philips Healthcare) with an 8-channel head coil. DTI tractography was performed as follows, using the same methodology previously described by our group. ${ }^{17,20}$ DTI was performed using a single-shot, multislice, spin echo-echo planar sequence with the following attributes: diffusion sensitization $1300 \mathrm{sec} / \mathrm{mm}^{2}$, TR $9577 \mathrm{msec}$, TE $77 \mathrm{msec}$, voxel size $2 \mathrm{~mm}^{3}$, no gap between slices, and matrix $224 \times 224$. Sixty-four diffusion gradient directions were obtained. The DTI data sets and anatomical MRI scans were analyzed with Fiber Track software from MR Workspace (Philips Healthcare) for diffusion tensor analysis and fiber tracking. To increase the number of detectable fibers, we applied a probabilistic tracking algorithm with progressive lowering of the fractional anisotropy value. A knowledge-based multiple region of interest (ROI) approach was applied, in which the tracking algorithm was initiated from user-defined seed regions. Axonal projections were traced in both anterograde and retrograde directions according to the direction of the principal eigenvector in each voxel of the region of interest. Tracking terminated when the fractional anisotropy value was $<0.18$.

The seed regions used to reconstruct the A-Arc, MLF, IFOF, and OR were placed according to previous descriptions by our group and other groups. ${ }^{5,17}$ The fiber tracking of the acoustic radiation (AR) was performed by using a 2-ROI approach previously described by Javad et al., ${ }^{10}$ in which the first ROI was placed in the white matter of Heschl's gyrus and the target ROI was set in the middle geniculate body, and both ROIs were identified in the anatomical T1-weighted MRI sequence. Outside the AR, a set of ROIs was chosen to avoid errant fiber trajectories. Fiber tracts passing through the starting and target points, but not through the excluded regions, were kept as the AR.

\section{Surgical Technique}

Six surgical patients with left temporal gliomas close to the HGFIA were included for the analysis. These patients were selected according to the following inclusion criteria: 1) age $>18$ years, 2) tumor located within or in proximity to the HGFIA, 3) intraoperative electrical stimulation mapping with an asleep-awake-asleep surgical technique ${ }^{19}$ (all surgeries were performed by the same author, J.M., between 2011 and 2014), and 4) histological confirmation of diffuse low-grade glioma WHO grade II-III. For the surgical results, a neuronavigation system was used to correlate the functional areas with the preoperative DTI tractography. The limits of the resection were defined depending on the eloquent regions identified in the surgical cavity.

All participants provided signed informed consent to participate in the research; all procedures were approved by the Hospital Universitario Marqués de Valdecilla Committee of Human Research (protocol number 2019.117), and all research procedures were directed according to the Declaration of Helsinki.

\section{Statistical Analysis}

The side of the hemisphere (right vs left) was selected as the independent variable. Meanwhile, the distances measured at Heschl's gyrus and the HGFIA and the identification or not of the different tracts at the HGFIA were chosen as independent variables. The Mann-Whitney Utest was used for quantitative variables, and Fisher's exact test was used for qualitative variables. In all cases the accepted significance level was 5\% ( $\mathrm{p}<0.05)$. The analysis was performed with SPSS software version 20.0 (IBM). 
TABLE 1. Tracts identified and distances measured at the HGFIA

\begin{tabular}{|c|c|c|c|c|c|c|c|c|c|c|c|}
\hline \multirow{2}{*}{$\begin{array}{l}\text { Hemisphere } \\
\text { No. }\end{array}$} & \multirow[b]{2}{*}{ Side } & \multirow{2}{*}{$\begin{array}{l}\text { FD vs } \\
\text { DTI }\end{array}$} & \multicolumn{6}{|c|}{ HGFIA Tracts } & \multicolumn{3}{|c|}{ HGFIA Distance (mm) } \\
\hline & & & A-Arc & MLF & AR & IFOF & OR & U Fibers & D1 & $\mathrm{D} 2$ & D3 \\
\hline 1 & $\mathrm{Lt}$ & FD & Yes & Yes & Yes & Yes & Yes & Yes & 51 & 82 & 53 \\
\hline 2 & Rt & FD & Yes & Yes & Yes & Yes & Yes & Yes & 46 & 74 & 47 \\
\hline 3 & $\mathrm{Lt}$ & FD & Yes & Yes & Yes & Yes & Yes & Yes & 53 & 84 & 55 \\
\hline 4 & Rt & FD & Yes & Yes & Yes & Yes & Yes & Yes & 50 & 75 & 56 \\
\hline 5 & $\mathrm{Lt}$ & FD & Yes & Yes & Yes & Yes & Yes & Yes & 47 & 76 & 55 \\
\hline 6 & Rt & FD & Yes & Yes & Yes & Yes & Yes & Yes & 45 & 72 & 42 \\
\hline 7 & $\mathrm{Lt}$ & FD & Yes & Yes & Yes & Yes & Yes & Yes & - & - & 46 \\
\hline 8 & $\mathrm{Rt}$ & FD & Yes & Yes & Yes & Yes & Yes & Yes & 52 & 77 & 55 \\
\hline 9 & $\mathrm{Lt}$ & DTI & Yes & Yes & Yes & Yes & Yes & Yes & 51 & 76.9 & 55.6 \\
\hline 10 & $\mathrm{Rt}$ & DTI & Yes & Yes & Yes & Yes & Yes & Yes & 52 & 79 & 50.3 \\
\hline 11 & $\mathrm{Lt}$ & DTI & Yes & Yes & Yes & Yes & Yes & Yes & 51.3 & 77.9 & 54.1 \\
\hline 12 & Rt & DTI & Yes & Yes & Yes & Yes & Yes & Yes & 51.8 & 77.4 & 53.6 \\
\hline 13 & $\mathrm{Lt}$ & DTI & Yes & Yes & Yes & Yes & Yes & Yes & 50 & 79 & 53.2 \\
\hline 14 & $\mathrm{Rt}$ & DTI & Yes & Yes & Yes & Yes & Yes & Yes & 52.3 & 80.6 & 53.8 \\
\hline 15 & $\mathrm{Lt}$ & DTI & Yes & Yes & Yes & Yes & Yes & Yes & 45.5 & 79 & 49.9 \\
\hline 16 & $\mathrm{Rt}$ & DTI & Yes & Yes & Yes & Yes & Yes & Yes & 50.2 & 76.3 & 48.5 \\
\hline Total & $\begin{array}{l}50 \% \mathrm{Lt} \\
50 \% \mathrm{Rt}\end{array}$ & $\begin{array}{l}50 \% \text { FD } \\
50 \% \text { DTI }\end{array}$ & $100 \%$ & $100 \%$ & $100 \%$ & $100 \%$ & $100 \%$ & $100 \%$ & $\begin{array}{l}\text { Mean } 49.87, \\
\quad \text { range } 45-53\end{array}$ & $\begin{array}{l}\text { Mean } 77.74, \\
\quad \text { range } 72-84\end{array}$ & $\begin{array}{l}\text { Mean } 51.75, \\
\quad \text { range } 42-56\end{array}$ \\
\hline
\end{tabular}

$\mathrm{FD}=$ fiber dissection; $-=$ measurement could not be obtained because the specimen was missing the temporal pole.

FD performed in 8 cadaveric specimens; DTI tractography performed in 4 healthy volunteers.

\section{Results}

The tracts identified at the HGFIA and the distances measured in each hemisphere are detailed in Table 1.

\section{Tracts Identified and Their Organization at the HGFIA}

Five white matter associative tracts were identified at the HGFIA (Figs. 1-3).

1. AR. These fibers originate from the middle geniculate body and project to the ipsilateral primary auditory cortex in Heschl's gyrus.

2. A-Arc. These fibers have an anteroposterior orientation and run lateral to the long segment of the L-Arc connecting the inferior parietal lobe and posterior temporal lobe to the ventral part of the precentral gyrus.

3. MLF. This pathway, running within the STG with an anteroposterior and superior orientation, connects the STG with the parietal lobe.

4. IFOF. This pathway runs within the frontal lobe, insula, temporal stem, and sagittal stratum and connects the frontal operculum with the occipital, parietal, and temporobasal cortex.

5. OR. These tracts originate from the lateral geniculate body and project to the ipsilateral primary visual cortex in the middle temporal gyrus (MTG), medial to the IFOF, and form the roof of the temporal horn.

In addition, in all hemispheres dissected and studied by tractography, $U$ fibers from the inferior segment of the STG to the MTG were also identified. These fibers curve around the superior temporal sulcus and are connected to the L-Arc at their termination.

\section{Tract Organization Within the HGFIA}

The associative tracts crossing the HGFIA are anatomically organized into 2 different layers. The superficial layer includes the AR, MLF, and A-Arc, and the U fibers connecting Heschl's gyrus with the MTG. The deep layer of tracts is formed by the IFOF and the OR.

\section{Superficial Layer}

Within the superficial layer (Fig. 3B), the tracts are intricately arranged from lateral to medial in the following order: A-Arc, AR, and MLF. In a similar way, the cortical projections of these tracts are organized from anterior to posterior as follows: 1) the MLF terminates anteriorly at the STG, 2) the AR terminates at Heschl's gyrus, and 3) more posteriorly, the A-Arc terminates at the supramarginal gyrus and the more posterior part of the STG (Fig. 3A).

The fibers of the AR, from lateral to medial, have a superior and posterior orientation. Medially, they converge at the caudal end of the insula, and laterally, at Heschl's gyrus, the fibers unfold in a fan.

The MLF is differentiated from the A-Arc by its deeper location and its anteroposterior and superior orientation. At the HGFIA, the fibers of the MLF terminate more anteriorly at the STG than the fibers of the AR (Figs. 1 and 2).

In addition to the associative tracts within the superficial layer of the HGFIA, U fibers connecting Heschl's gyrus with the MTG were identified in both the DTI-tractography and the fiber dissection (Figs. 2, 3B, and 3C).

\section{Deep Layer}

The deep layer of the HGFIA (Fig. 3C) includes the 


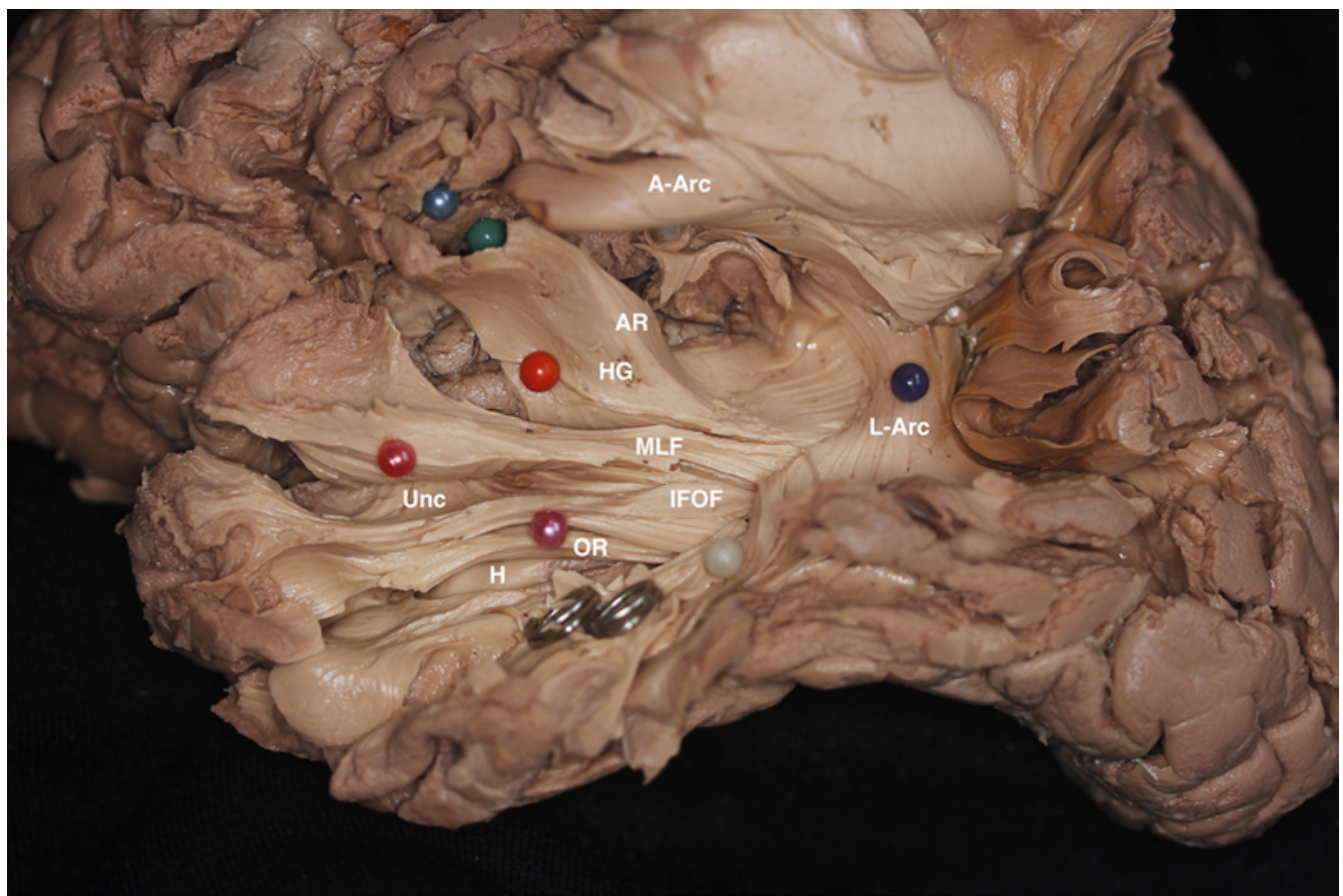

FIG. 1. Fiber dissection of the tracts identified at the HGFIA in a left hemisphere. Heschl's gyrus and the A-Arc have been tilted superiorly to expose the MLF, L-Arc, and IFOF. The dissection demonstrates that 5 white matter tracts cross the HGFIA, the A-Arc, MLF, AR, IFOF, and OR. These tracts are anatomically organized in 2 different layers. The most superficial layer includes the AR, $\mathrm{LF}$, and A-Arc (arranged from anterior to posterior), whereas the IFOF and the OR are located in the deeper layer. $\mathrm{H}=$ temporal horn; HG = Heschl's gyrus; OR = optic radiation; Unc = uncinate fasciculus.

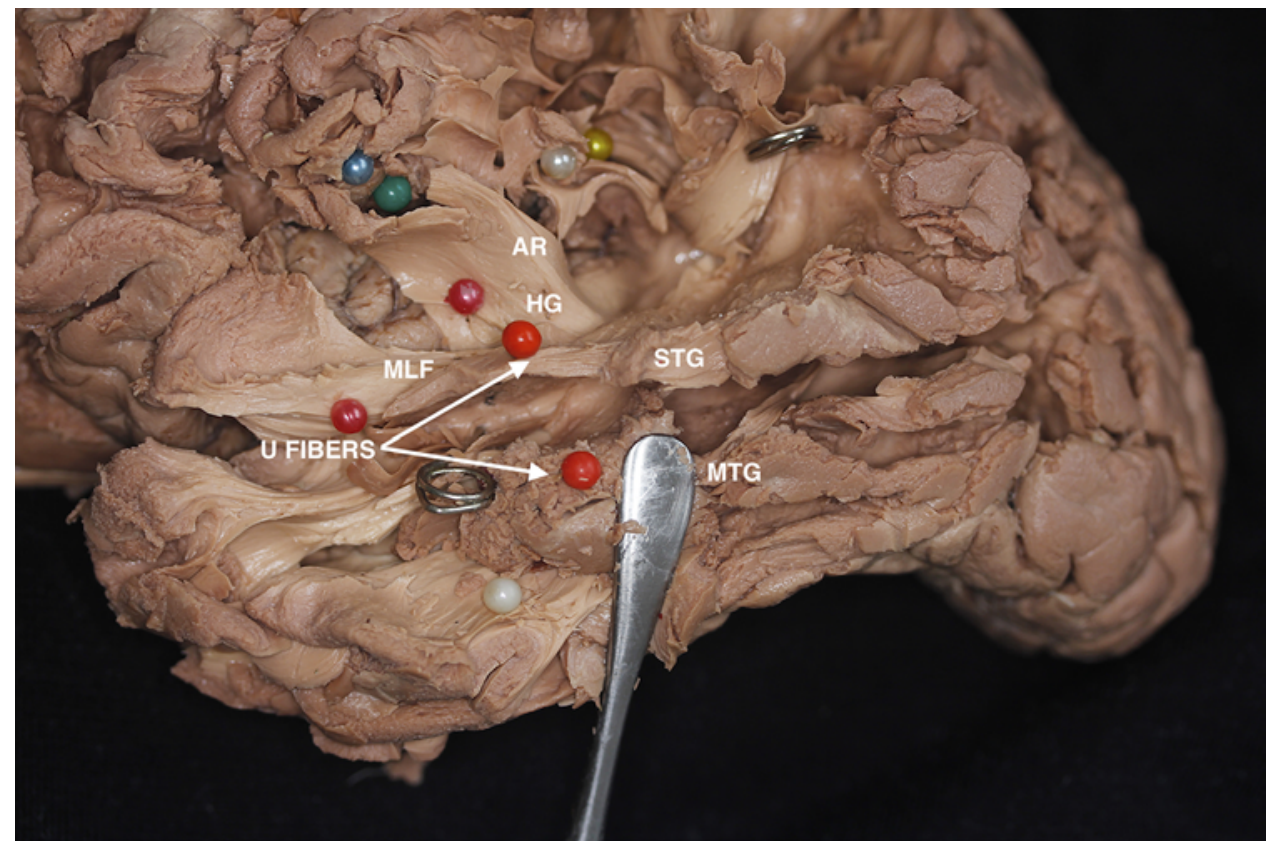

FIG. 2. Fiber dissection of the tracts identified at Heschl's gyrus in a left hemisphere. Heschl's gyrus has been tilted superiorly to expose the MLF. The dissection demonstrates that Heschl's gyrus is connected with the MTG through some U-fiber bundles. The dissection demonstrates the MLF fibers ending more anteriorly than the AR in the STG and some fibers originating more anteriorly in the STG and coursing posteriorly and deeper toward the MTG. HG = Heschl's gyrus. 


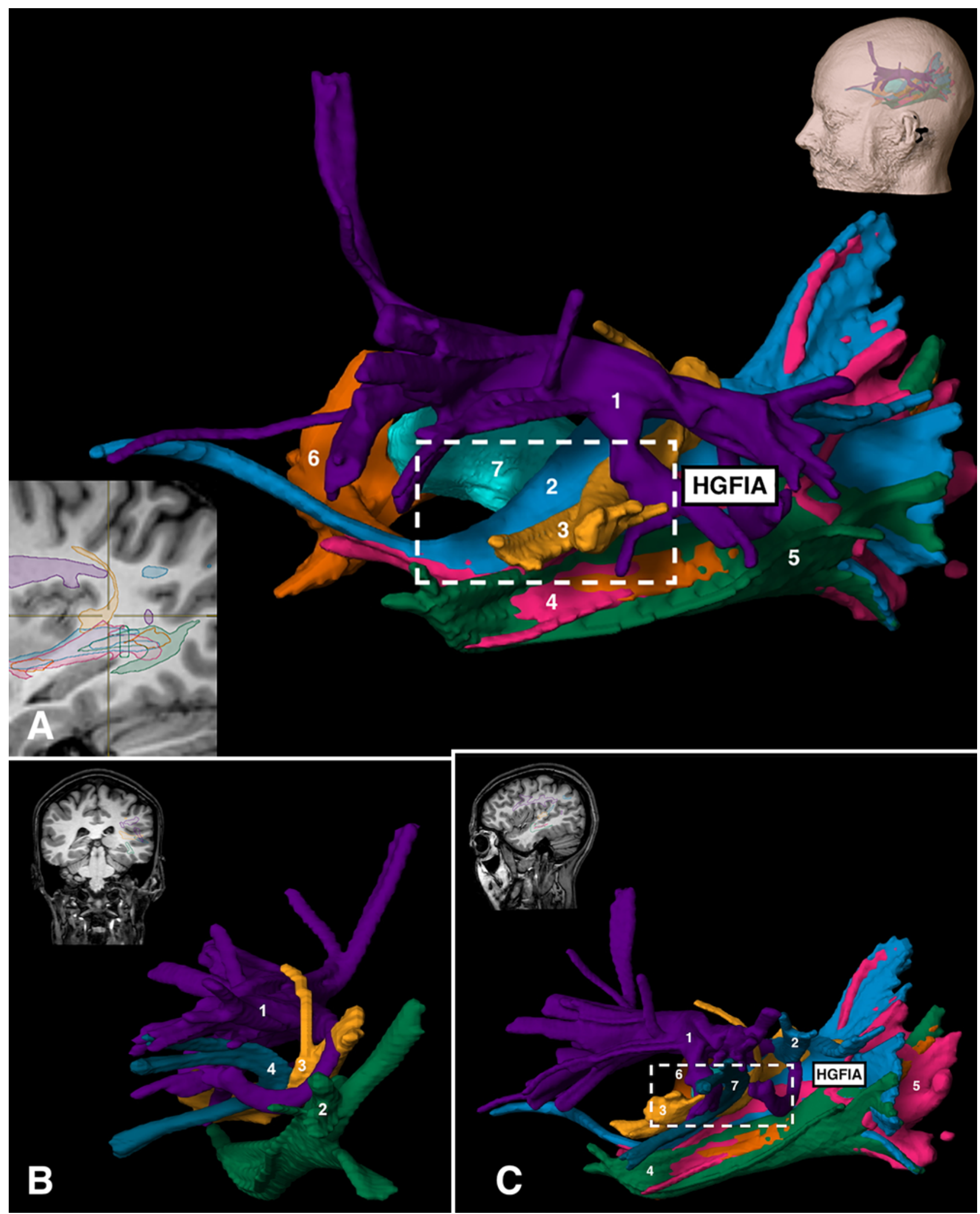

FIG. 3. A: DTI tractography 3D reconstruction of the tracts that cross at the HGFIA in a left hemisphere (white dashed rectangle). $1=\mathrm{A}-\mathrm{Arc} ; 2=\mathrm{MLF} ; 3=\mathrm{AR} ; 4=\mathrm{IFOF} ; 5=\mathrm{OR} ; 6=$ anterior comissure; $7=$ left thalamus. B: DTI tractography 3D reconstruction of the tracts identified at Heschl's gyrus in a left hemisphere and the connections between them. $1=\mathrm{A}-\operatorname{Arc} ; 2=\mathrm{MLF} ; 3=\mathrm{AR} ; 4=\mathrm{U}$ fibers. C: DTI tractography $3 \mathrm{D}$ reconstruction of the tracts that cross at the HGFIA in a left hemisphere (white dashed rectangle). 1 $=\mathrm{A}-\mathrm{Arc} ; 2$ = MLF; 3 = AR; 4 = IFOF; 5 = OR; 6 = anterior comissure; 7 = U fibers. 


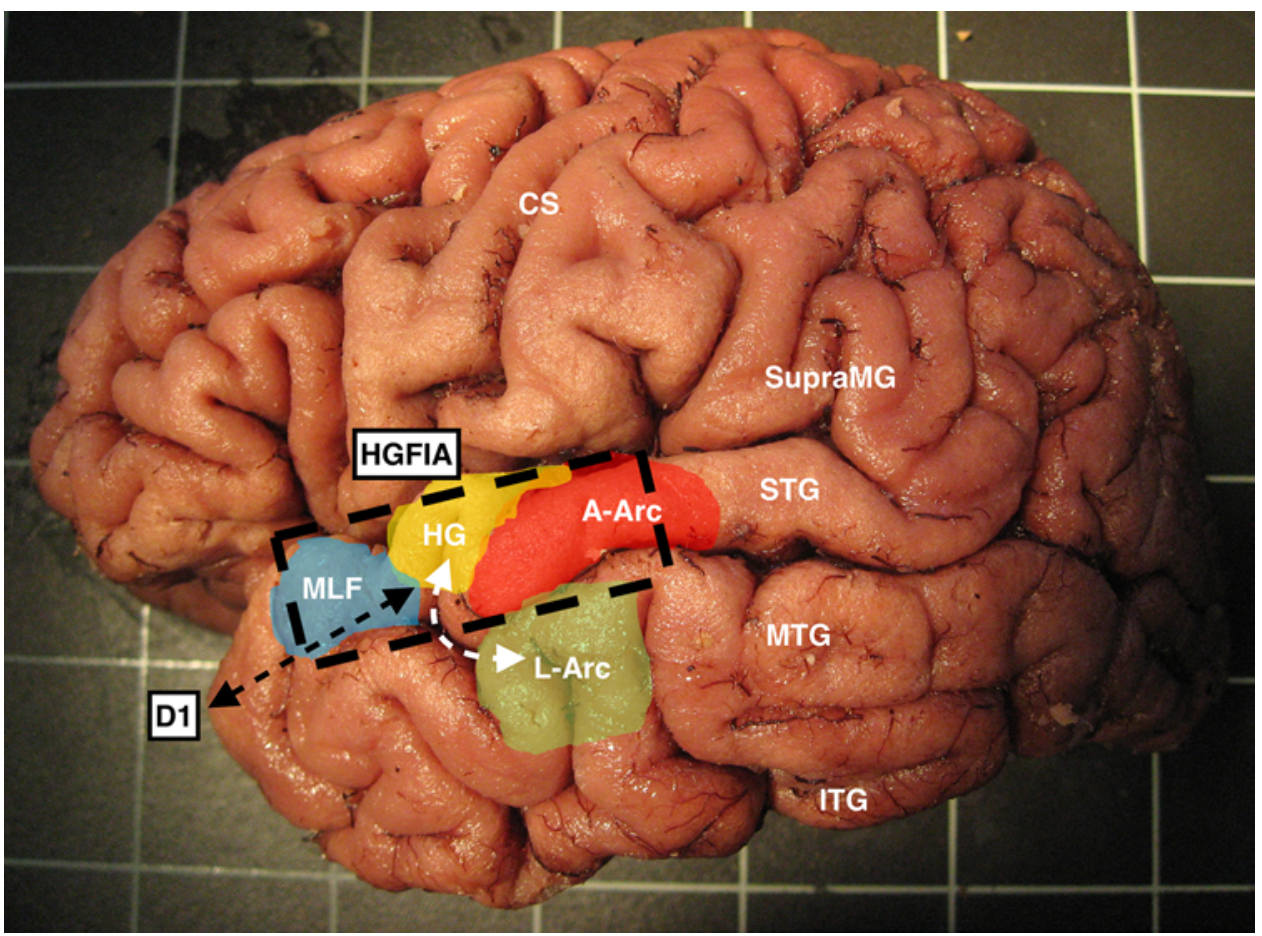

FIG. 4. Schematic representation depicting the location of Heschl's gyrus, the cortical projections of the main tracts crossing the HGFIA (black dashed rectangle), the distance D1 (black dashed line with arrowheads) between the temporal pole and the anterior limit of Heschl's gyrus at the convexity surface of the hemisphere (average $49.87 \mathrm{~mm}$, range $45-53 \mathrm{~mm}$ ), and a U-fiber bundle (white dashed line with arrowheads). CS = central sulcus; HG = Heschl's gyrus; ITG = inferior temporal gyrus; SupraMG = supramarginal gyrus.

IFOF superiorly and the OR inferiorly (Figs. 1 and 3A). The IFOF crosses under the A-Arc in an anterior-posterior and superior direction. Similarly, the OR runs medial to the IFOF, following the same orientation, and is located above the fibers of the lateral limb of the anterior commissure and tapetum, forming the roof of the temporal horn and atrium.

\section{Distances Measured}

The following 3 distances were measured at the HGFIA (Table 1, Fig. 4):

1. D1: The shortest distance between the temporal pole and the anterior limit of Heschl's gyrus at the convexity surface of the hemisphere (average $49.87 \mathrm{~mm}$, range, $45-53 \mathrm{~mm}$ ).

2. D2: The shortest distance between the temporal pole and the anterior limit of Heschl's gyrus at the inferior limiting sulcus of the insula (average $77.74 \mathrm{~mm}$, range $72-84 \mathrm{~mm}$ ).

3. D3: The shortest distance between the limen insulae and Heschl's gyrus at the inferior limiting sulcus of the insula (average $51.75 \mathrm{~mm}$, range $42-56 \mathrm{~mm}$ ).

These 3 distances were compared between both hemispheres. The differences were not statistically significant $(\mathrm{p}>0.05)$.

\section{Surgical Findings}

Cortical electrical stimulation at the HGFIA elicited language disorders in all patients (Fig. 5). Anomia presented in 4 patients, in 1 patient at 3 cortical areas and in 4 patients at 1 cortical area. Semantic paraphasia was found in 4 patients at 1 cortical area.

During subcortical stimulation, language disorders were detected in 4 patients (Fig. 5). Anomia was elicited in 3 patients, and semantic paraphasia in 1 patient.

The histopathological analysis revealed diffuse infiltrative astrocytoma in 4 patients, anaplastic glioma in 1 patient, and glioblastoma in 1 patient.

To visually depict the functional anatomy of the HGFIA, pre- and intraoperative images for 2 illustrative cases are presented: a patient with diffuse infiltrative astrocytoma (case 1; Fig. 6) and a patient with an anaplastic astrocytoma (case 2; Fig. 7).

\section{Discussion}

\section{HGFIA Organization and New Insights on Language Processing}

The unique trajectory of the AR from the middle geniculate body to the cortex of Heschl's gyrus is well described, but the relationship of these structures with the adjacent language pathways has been analyzed in only a few previous works., ${ }^{2,13,21}$ Moreover, the complex tract organization within the HGFIA could be considered the anatomical substrate of the functional connection between the primary auditory cortex, the endpoint of the AR, and the dorsal language stream, and hence to other language net- 



FIG. 5. A-F: Schematic representation of the 6 surgical cases presented. In all cases the cortical and subcortical stimulation in the HGFIA elicited language disorders (anomia or semantic paraphasia). White dotted line areas indicate cortical (posterior part of the STG) and subcortical (HGFIA) areas where language disorders were elicited (Spanish flags indicate areas where language function was detected).

works. Based on our findings, the primary auditory area is at the center of a region encircled by 3 important connections: 1) inferiorly, by $U$ fibers that connect the primary auditory area with the MTG, and then to the L-Arc; 2) posteriorly, by the A-Arc that connects the posterior portion of the STG with the ventral premotor cortex; and 3) 

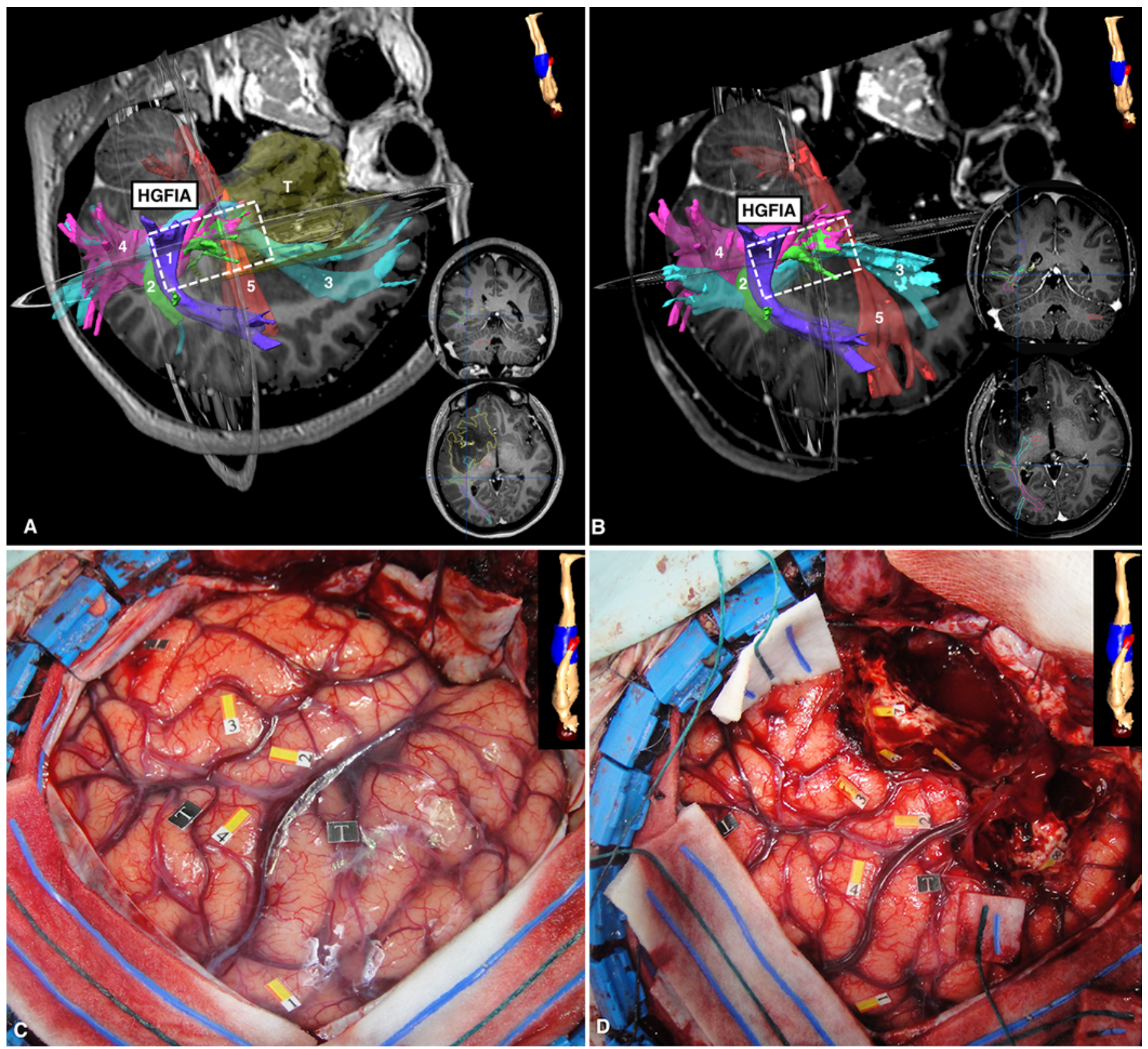

FIG. 6. Images of case 1, a 48-year-old, monolingual, right-handed woman with a left frontotemporal diffuse infiltrative astrocytoma (representation of patient position for orientation shown in upper right corner of A-D). The patient had smelling-type partial seizures and partial seizures with aphasia 2 months before admission. No preoperative deficits were observed. A: Preoperative sagittal FLAIR MRI with DTI tractography reconstruction of the tracts ending at the HGFIA (white dashed rectangle). The tumor at the temporal lobe is anterior to the HGFIA, in direct contact with Heschl's gyrus and the MLF, A-Arc, IFOF, and the OR. $1=$ A-Arc; $2=\mathrm{MLF} ; 3=\mathrm{IFOF} ; 4=\mathrm{OR} ; 5=$ pyramidal pathway; $\mathrm{T}=$ tumor. B: Postoperative sagittal FLAIR MRI with DTI tractography reconstruction of the tracts ending at the HGFIA (white dashed rectangle). $1=\mathrm{A}-\mathrm{Arc} ; 2=\mathrm{MLF} ; 3=\mathrm{IFOF} ; 4=\mathrm{OR} ; 5=$ pyramidal pathway. C: Intraoperative photograph before tumor resection exposing the left frontal and temporal lobes. Intraoperative cortical electrical stimulation at the posterior part of the STG elicited anomia. This area corresponding to the HGFIA was located with neuronavigation. Intraoperative cortical electrical stimulation elicited the following responses: Spanish flag $1=$ speech arrest at the premotor lateral ventral cortex; Spanish flags 2 and $3=$ anomia at the posterior part of the STG and MTG; Spanish flag $4=$ anarthria at the inferior part of the postcentral circumvolution. T = tumor. D: Intraoperative photograph after tumor resection. The limits of the resection were defined depending on the eloquent regions identified in the surgical cavity. Intraoperative subcortical electrical stimulation elicited the following responses: Spanish flags 5-7 = anomia; Spanish flag $8=$ language difficulties with perseveration because of the resection near to the caudate nucleus head.

anteriorly, by the MLF that connects the middle and anterior portions of the STG with the parietal lobe.

Auditory feedback is an essential component of speech articulation. In fact, ischemic lesions of Heschl's gyrus white matter result in impaired feedback connectivity to auditory areas, which may cause speech comprehension 

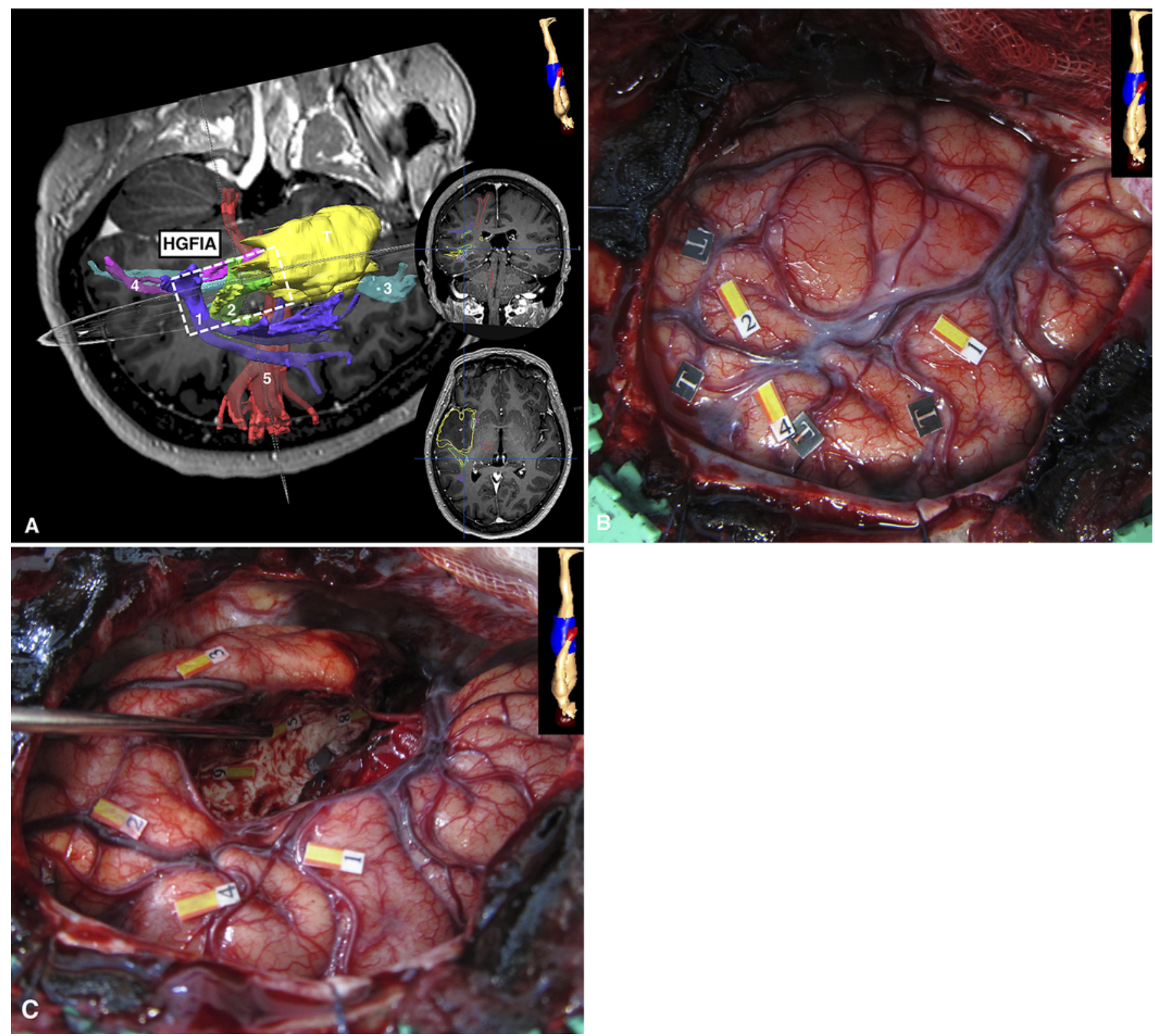

FIG. 7. Images of case 2, a 59-year-old, monolingual, right-handed woman with a temporoinsular anaplastic WHO grade III astrocytoma. The patient had an isolated partial seizure with aphasia 1 month before admission. No preoperative deficit was observed (representation of patient position for orientation shown in upper right corner of A-C). A: Preoperative sagittal FLAIR MRI with DTI tractography reconstruction of the tracts ending at the HGFIA (white dashed rectangle). The tumor at the temporal lobe is anterior to the HGFIA, in direct contact with Heschl's gyrus and the MLS, the A-Arc, the IFOF, and the OR. 1 = A-Arc; 2 = MLF; $3=$ IFOF; $4=\mathrm{OR} ; 5$ = pyramidal pathway. B: Intraoperative photograph before tumor resection exposing the left frontal and temporal lobes. Intraoperative cortical electrical stimulation at the posterior part of the STG elicited anomia and semantic paraphasia. This area, corresponding to the HGFIA, was located with neuronavigation. Intraoperative cortical electrical stimulation elicited the following responses: Spanish flag $1=$ speech arrest at the premotor lateral ventral cortex; Spanish flag $2=$ anomia at the posterior part of the STG; Spanish flag 3 = anomia at the MTG; Spanish flag $4=$ syntax disorder in the task of lecture at the inferior part of the postcentral circumvolution. T = tumor. C: Intraoperative photograph after tumor resection. Spanish flags 1-4 = responses as described for panel $\mathrm{C}$. The limits of the resection were defined according to the eloquent regions identified in the surgical cavity. Intraoperative subcortical electrical stimulation elicited the following responses: Spanish flags 5, 6, and $8=$ anomia.

difficulties. ${ }^{26}$ The voice is simultaneously produced in the mouth and heard at the primary auditory cortex. This auditory-motor loop is essential to constantly monitor and correct speech output. ${ }^{9,11,12}$ The dysfunction of this loop produces the articular disorder that is observed in deaf in- dividuals. The present work supports the hypothesis that the primary auditory area is in direct contact posteriorly with the A-Arc. The A-Arc represents a direct and rapid connection between the temporal lobe and the speech output area at the ventral premotor cortex..$^{24,25}$ Therefore, this 
connection represents a very suitable route to explain the articulatory loop. In sum, our findings may support the hypothesis that the voice is produced in the mouth and simultaneously heard through the auditory system (external loop). Then, from the primary auditory cortex the information may be rapidly sent posteriorly to the cortex connected to the A-Arc. Finally, the A-Arc sends it to the speech output area for precise monitoring of speech articulation (internal loop).

The L-Arc is considered an essential network for phonological integration, as it plays a key role in the linkage between the acoustic sensorial input and speech production..$^{3,7}$ In fact, intraoperative electrical stimulation of this connection induces phonemic paraphasias with high reproducibility. In a phonemic paraphasia, the target word is correct, but the phonemes are transpositioned or substituted, 7,25 for example, "blate" instead of "table." In the present work, we identified U fibers that connect the STG with the MTG. These U fibers may represent a direct connection between the primary auditory area and the arcuate fascicle and would explain the integration of the auditory entrance into the MTG, and from there to the ventral premotor cortex and the posterior segment of the lower frontal gyrus through the L-Arc.

The last superficial connection related to Heschl's gyrus is the MLF. The MLF connects the cortex immediately anterior to the primary auditory area to the parietal lobe. ${ }^{14}$ The functional role of this connection is much more speculative. The parietal lobe is important to sensorimotor and visual integration; therefore, this connection between the auditory and parietal cortex may be important to localize the source of a sound. ${ }^{8,22,25}$ Further connection with the eye movement control area would cause saccadic movement of the eyes toward the source. This connection may also be important for the verbal working memory circuit. The frontoparietal networks are essential for retaining words in memory and manipulating verbal information. After a word is heard, the MLF may send the information to the parietal lobe, where the working memory network keeps firing through the delay period in which there is no stimulus, in order to temporarily hold the information in memory. ${ }^{24,25}$ For example, imagine that you are at a scientific meeting, and you hear in a lecture the unusual word "scarecrow." Five hours later you hear that word again, and you remember that you heard the word during the lecture.

\section{Surgical Implications}

Intraoperative electrical stimulation of the HGFIA elicited language disturbances in all cases at the cortical and subcortical level. These results emphasize the important functional role of this area, which must be identified and preserved during surgery. It is important to note that resection of the STG, anterior to Heschl's gyrus, did not result in a permanent language deficit in any of the study patients, despite the resection of a large part of the MLF. This result is in line with the hypothesis that the MLF in humans is not essential for language processing. ${ }^{6}$ Moreover, the distances measured in the laboratory dissections and tractography analyses are in accordance with the posterior limit of the resection in the left temporal approaches, because the function was detected posterior to the connection of the MLF.

The HGFIA is also associated with other tracts that are not directly connected to it, but that pass through the deep white matter. Within this deep layer of tracts, the IFOF and the OR are highly eloquent and should also be preserved. In the cases described here, semantic paraphasias were elicited during subcortical electrical stimulation at the deepest level, in line with the hypothesis of the IFOF as an essential component of the semantic language system. Therefore, the HGFIA is related to the 2 streams of language, superficially to the dorsal phonological stream, as it is connected to the L-Arc and A-Arc, and deeply to the ventral semantic stream, as it is related to the IFOF.

\section{Limitations}

The results presented here have the following limitations. First, the potential variability of the connectivity of the HGFIA cannot be evaluated on the basis of our results because of the small number of study patients. Second, it is well known that fiber dissection techniques and DTI tractography are limited methods to analyze white fiber intersection areas. Finally, the intraoperative electrical stimulation mapping to evaluate function was limited due to the current dispersion in tract intersection areas. Consequently, further studies are necessary to analyze in more detail the connectivity and the function of this region.

\section{Conclusions}

This anatomical study delineated the HGFIA, a region where 5 fiber tracts intersect in a relationship with the primary auditory area. The HGFIA is a key region in the language system, allowing the integration of auditory input and speech production. Our findings support the hypothesis that the output of information at the level of the auditory cortex is sent via the arcuate fasciculus complex to the speech output areas through 2 pathways, indirectly through the small $\mathrm{U}$ fibers connecting the auditory area with the MTG and the L-Arc and directly through the AArc. In addition, the auditory input is also integrated in the parietal association cortex via the MLF. Moreover, the HGFIA is also intimately related to the ventral semantic stream, as the IFOF crosses through the deep white matter.

\section{References}

1. Binder JR: The Wernicke area: modern evidence and a reinterpretation. Neurology 85:2170-2175, 2015

2. Bürgel U, Amunts K, Hoemke L, Mohlberg H, Gilsbach JM, Zilles K: White matter fiber tracts of the human brain: threedimensional mapping at microscopic resolution, topography and intersubject variability. Neuroimage 29:1092-1105, 2006

3. Catani M, Jones DK, ffytche DH: Perisylvian language networks of the human brain. Ann Neurol 57:8-16, 2005

4. Catani M, Mesulam M: The arcuate fasciculus and the disconnection theme in language and aphasia: history and current state. Cortex 44:953-961, 2008

5. Catani M, Thiebaut de Schotten M: A diffusion tensor imaging tractography atlas for virtual in vivo dissections. Cortex 44:1105-1132, 2008

6. De Witt Hamer PC, Moritz-Gasser S, Gatignol P, Duffau 
$\mathrm{H}$ : Is the human left middle longitudinal fascicle essential for language? A brain electrostimulation study. Hum Brain Mapp 32:962-973, 2011

7. Duffau H, Moritz-Gasser S, Mandonnet E: A re-examination of neural basis of language processing: proposal of a dynamic hodotopical model from data provided by brain stimulation mapping during picture naming. Brain Lang 131:1-10, 2014

8. Friederici AD: The brain basis of language processing: from structure to function. Physiol Rev 91:1357-1392, 2011

9. Hickok G, Poeppel D: Dorsal and ventral streams: a framework for understanding aspects of the functional anatomy of language. Cognition 92:67-99, 2004

10. Javad F, Warren JD, Micallef C, Thornton JS, Golay X, Yousry T, et al: Auditory tracts identified with combined fMRI and diffusion tractography. Neuroimage 84:562-574, 2014

11. Liberman AM, Cooper FS, Shankweiler DP, StuddertKennedy M: Perception of the speech code. Psychol Rev 74:431-461, 1967

12. Liberman AM, Mattingly IG: The motor theory of speech perception revised. Cognition 21:1-36, 1985

13. Maffei C, Jovicich J, De Benedictis A, Corsini F, Barbareschi M, Chioffi F, et al: Topography of the human acoustic radiation as revealed by ex vivo fibers micro-dissection and in vivo diffusion-based tractography. Brain Struct Funct 223:449-459, 2018

14. Makris N, Papadimitriou GM, Kaiser JR, Sorg S, Kennedy DN, Pandya DN: Delineation of the middle longitudinal fascicle in humans: a quantitative, in vivo, DT-MRI study. Cereb Cortex 19:777-785, 2009

15. Martino J, Brogna C, Robles SG, Vergani F, Duffau H: Anatomic dissection of the inferior fronto-occipital fasciculus revisited in the lights of brain stimulation data. Cortex 46:691-699, 2010

16. Martino J, da Silva-Freitas R, Caballero H, Marco de Lucas E, Garcia-Porrero JA, Vazquez-Barquero A. Fiber dissection and diffusion tensor imaging tractography study of the temporoparietal fiber intersection area. Neurosurgery 72(1 Suppl Operative):ons87-ons98, 2013

17. Martino J, De Witt Hamer PC, Berger MS, Lawton MT, Arnold CM, de Lucas EM, et al: Analysis of the subcomponents and cortical terminations of the perisylvian superior longitudinal fasciculus: a fiber dissection and DTI tractography study. Brain Struct Funct 218:105-121, 2013

18. Martino J, De Witt Hamer PC, Vergani F, Brogna C, de Lucas EM, Vázquez-Barquero A, et al: Cortex-sparing fiber dissection: an improved method for the study of white matter anatomy in the human brain. J Anat 219:531-541, 2011

19. Martino J, Mato D, Marco de Lucas E, García-Porrero JA, Gabarrós A, Fernández-Coello A, et al: Subcortical anatomy as an anatomical and functional landmark in insulo-opercular gliomas: implications for surgical approach to the insular region. J Neurosurg 123:1081-1092, 2015

20. Martino J, Vergani F, Robles SG, Duffau H. New insights into the anatomic dissection of the temporal stem with special emphasis on the inferior fronto-occipital fasciculus: implications in surgical approach to left mesiotemporal and temporoinsular structures. Neurosurgery 66(3 Suppl Operative):4-12, 2010
21. Menjot de Champfleur N, Lima Maldonado I, Moritz-Gasser S, Machi P, Le Bars E, Bonafé A, et al: Middle longitudinal fasciculus delineation within language pathways: a diffusion tensor imaging study in human. Eur J Radiol 82:151-157, 2013

22. Rijntjes M, Weiller C, Bormann T, Musso M: The dual loop model: its relation to language and other modalities. Front Evol Neurosci 4:9, 2012

23. Sarubbo S, De Benedictis A, Merler S, Mandonnet E, Barbareschi M, Dallabona M, et al: Structural and functional integration between dorsal and ventral language streams as revealed by blunt dissection and direct electrical stimulation. Hum Brain Mapp 37:3858-3872, 2016

24. Saur D, Kreher B, Schnell S, Kümmerer D, Kellmeyer P, Vry MS, et al. Ventral and dorsal pathways for language. Proc Natl Acad Sci U S A 105:18035-18040, 2008

25. Saur D, Schelter B, Schnell S, Kratochvil D, Küpper H, Kellmeyer P, et al: Combining functional and anatomical connectivity reveals brain networks for auditory language comprehension. Neuroimage 49:3187-3197, 2010

26. Schofield TM, Penny WD, Stephan KE, Crinion JT, Thompson AJ, Price CJ, et al: Changes in auditory feedback connections determine the severity of speech processing deficits after stroke. J Neurosci 32:4260-4270, 2012

27. Tanriover N, Rhoton AL Jr, Kawashima M, Ulm AJ, Yasuda A: Microsurgical anatomy of the insula and the sylvian fissure. J Neurosurg 100:891-922, 2004

28. Wen HT, Rhoton AL Jr, de Oliveira E, Castro LHM, Figueiredo EG, Teixeira MJ: Microsurgical anatomy of the temporal lobe: part 2-sylvian fissure region and its clinical application. Neurosurgery 65 (6 Suppl):1-36, 2009

29. Wernicke C: The aphasic symptom-complex: a psychological study on an anatomical basis. Arch Neurol 22:280-282, 1970

\section{Disclosures}

The authors report no conflict of interest concerning the materials or methods used in this study or the findings specified in this paper.

\section{Author Contributions}

Conception and design: Martino, Fernández, Velásquez. Acquisition of data: all authors. Analysis and interpretation of data: Martino, Fernández, Velásquez, de Lucas. Drafting the article: Martino, Fernández, Velásquez. Critically revising the article: all authors. Reviewed submitted version of manuscript: all authors. Approved the final version of the manuscript on behalf of all authors: Martino. Statistical analysis: Fernández. Administrative/technical/material support: Martino, García Porrero, de Lucas. Study supervision: Martino.

\section{Correspondence}

Juan Martino: Hospital Universitario Marqués de Valdecilla and Fundación Instituto de Investigación Marqués de Valdecilla, Santander, Cantabria, Spain. juan.martino@hotmail.com. 